\title{
VISA: Versatile Impulse Structure Approximation for Time-Domain Linear Macromodeling
}

\author{
Chi-Un Lei and Ngai Wong \\ Department of Electrical and Electronic Engineering \\ The University of Hong Kong, Pokfulam Road, Hong Kong \\ Email: \{culei,nwong\}@eee.hku.hk
}

\begin{abstract}
We develop a rational function macromodeling algorithm named VISA (Versatile Impulse Structure Approximation) for macromodeling of system responses with (discrete) time-sampled data. The ideas of Walsh theorem and complementary signal are introduced to convert the macromodeling problem into a non-pole-based Steiglitz-McBride (SM) iteration (a class of first- and second-order interpolations) without initial guess and eigenvalue computation. We demonstrate the fast convergence and the versatile macromodeling requirement adoption through a $P$-norm approximation expansion, using examples from practical data.
\end{abstract}

\section{INTRODUCTION}

In deep-submicron VLSI design, signal integrity analysis constantly requires efficient modeling and high-frequency simulation of passive structures such as packages and interconnect networks [1]. As full-wave electromagnetic (EM) analysis over a global system is generally impractical, data-driven rational function approximation algorithms, such as Vector Fitting (VF) etc. [2], have been used to construct reduced-order models from measurement or EM simulation for efficient simulation. Due to its high computational cost, the full-wave analysis is usually terminated before all transient responses vanish so that truncated time responses are obtained. Accurate macromodeling from truncated time-sampled data is therefore desirable.

Subspace-based State-Space System Identification (4SID) techniques [3] and Generalized Pencil-Of-Function (GPOF) methods [4] have been used for identification of linear structures with time-sampled data. But these methods are based on large-scale matrix operations and expensive singular value decomposition (SVD), and are less practical for fast and multiport macromodeling. VF-related techniques such as TimeDomain Vector Fitting (TD-VF) [5, 6] and Discrete-Time Domain Vector Fitting (TD-VFz) [7] have been developed for macromodeling of time-sampled data with less computational cost. Also, multiple-input multiple-output (MIMO) TD-VF has been applied for package macromodeling [5] and circuit extraction [6]. However, their performance is limited by the pole-basis calculation, eigenvalue computation and distortion due to nonlinear pole flipping. In light of the discrete-time nature of the time-sampled response, a least-squares single- input single-output macromodeling technique (SISO LS) has been proposed recently [8]. The idea is to regard the system response as a finite-length discrete response sequence of a finite-impulse-response (FIR) filter, and then a least-squares (LS) infinite-impulse-response (IIR) filter [9], usually of much lower order, is used to approximate the FIR response. This (low-order) IIR filter then provides a rational function capture of the macromodel. Compared to VF-based algorithms, SISO LS avoids the numerically sensitive eigenvalue computation, nonlinear pole flipping and initial pole assignment.

In this paper, we extend the original SISO LS and propose VISA (Versatile Impulse Structure Approximation) for efficient MIMO macromodeling. After the introduction in Section II, the mechanism of VISA is presented in Section III. The algorithm convergence analysis, convergence-related features (model order selection and $P$-norm approximation) and MIMO extension are shown in Sections IV and V, respectively. Macromodeling examples of real-world multiport data in Section VI then confirm the efficiency and accuracy of VISA.

\section{TIME-DOMAIN MACROMODELING}

Multiport linear macromodeling aims at modeling a linear multi-port structure with $p$ input ports and $q$ output ports, whose responses can be obtained by exciting one input port at a time and computing or measuring the responses at all output ports. Similar to (TD-)VF, a multi-port macromodel is often cast as a MIMO transfer matrix with a common denominator (poles) and a specific numerator (zeros) for each port response. Macromodeling techniques intend to fit the rational function

$\widehat{f}_{u, v}(s)=\frac{P_{u, v}(s)}{Q(s)}=\sum_{m=0}^{M} \frac{p_{u, v}(m) s^{m}}{q_{m} s^{m}}=d+\sum_{m=1}^{M} \frac{r_{u, v}(m)}{s-\alpha_{m}}$,

where $p_{u, v}(m), q(m) \in \Re, q(0)=1$, to the desired response $f_{u, v}(s)$ at a set of calculated/sampled points at input port $u(1 \leq u \leq p)$ and output port $v(1 \leq v \leq q)$. The data points can be frequency-sampled data (i.e., $f_{u, v}\left(s_{n}\right)$, for $n=1,2, \cdots, N_{s}$ ) or discrete-time-sampled data (i.e., input response $G_{u, v}[n]$ and output response $H_{u, v}[n]$, for $n=$ $0,1, \cdots, L-1)$. For ease of explanation, the SISO case is used in the following exposition. The extension to multiport macromodeling is presented in Section V. 
In time-domain macromodeling, TD-VF [2], a reformation of VF [2], attempts to fit the rational function (1) to a set of calculated/sampled data points at given time in the continuoustime domain. Starting with a set of $M$ prescribed or approximated poles $\left\{a_{m}^{(0)}\right\}, m=1,2, \cdots, M$, input response $x(t)$ and output response $y(t), t=0,1, \cdots, L-1$, the problem is linearized into a separable denominator calculation for the $k$ th iteration to find model parameters $d,\left\{c_{m}\right\},\left\{\gamma_{m}\right\}$, namely,

$$
y(t) \approx d x(t)+\sum_{m=1}^{M} c_{m} x_{m}^{(k)}(t)-\sum_{m=1}^{M} \gamma_{m} y_{m}^{(k)}(t),
$$

where $x_{m}^{(k)}(t)=\int_{0}^{t} e^{a_{m}^{(k)}(t-\tau)} x(\tau) d \tau, \quad y_{m}^{(k)}(t)=$ $\int_{0}^{t} e^{a_{m}^{(k)}(t-\tau)} y(\tau) d \tau, k=0,1, \cdots, N_{T}$, where $N_{T}$ denotes the number of iterations. $x_{m}^{(k)}(t)$ and $y_{m}^{(k)}(t)$ are integral convolutions, and obtained by a discretization process during each iteration. A discrete-time domain (i.e., z-domain) TD-VF, called TD-VFz, has been proposed recently [8], as a generalization from Discrete Time Vector Fitting, VFz [10,11]. As in TD-VF, TD-VFz uses discrete-time domain partial fractions to seek a rational approximation, $\widehat{F}(z)$, to the desired $z$-domain response $F(z)$ and applies only one discretization process after modeling. It is shown that the convergence is improved due to the reduction of discretization process. Furthermore, the numerical conditioning for broadband macromodeling is improved in the discrete-time domain, leading to a more accurate and faster converging modeling process [12].

\section{FORMULATION OF VISA}

Compared to existing algorithms, a promising observation in VISA is that an optimal numerator polynomial can be determined with respect to a given denominator polynomial, based on simple division algorithm, without numericalsensitive pole-finding calculations. The approximation problem boils down to finding a near-optimal and stable $Q(z)$. In the proposed algorithm, the obtained output response is assumed to be generated by a normalized input pulse response $(G[0]=1$ and $G[n]=0$ for $n=1,2, \cdots, L-1)$, in order to fit the response. This can be done by the deconvolution of the original output response with the input response.

\section{A. Numerator calculation}

First, we define the error term $\Delta(z)$ :

$$
\Delta(z)=H(z)-(P(z) / Q(z)),
$$

where $H(z)$ is specified as a FIR filter response approximation of a system. Computing $P(z) / Q(z)$ requires non-linear computations, but if the poles $\left(\alpha_{1}, \alpha_{2}, \ldots, \alpha_{M}\right)$ are given, based on the Walsh's Theorem [13], the best $L_{2}$ approximation to $H(z)$ is the unique function that interpolates to $H(z)$ in all the points, where $z=\infty, 1 / \alpha_{1}^{*}, 1 / \alpha_{2}^{*}, \ldots, 1 / \alpha_{M}^{*}$, and $*$ denotes complex conjugate. For the situation with no repeated poles, it means

$$
\left.H(z)\right|_{z=z_{k}}=P(z) /\left.Q(z)\right|_{z=z_{k}},
$$

where $k=0,1, \ldots M$, and (4) dictates that $\Delta(z)$ vanishes in the points $z_{k}$ [13]. Since the approximant is the impulse response approximation of a system, the interpolation condition (4) is used to describe $\Delta(z)$ by a cascade of a causal FIR filter $R(z)$ and an allpass filter $A(z)$ [14],

$$
\Delta(z)=A(z) z^{-1} R(z)=\frac{z^{-M} Q\left(z^{-1}\right)}{Q(z)} z^{-1} R(z),
$$

where $R(z)=\sum_{n=0}^{L-1} r_{n} z^{-n}$ and the zeros of $A(z)$ are the interpolation points $z_{k}$. With (3), the interpolation problem can be described as an input-output description of a digital filtering operation,

$$
\begin{gathered}
r_{L-1-n}=u_{n} \quad(n=0,1, \ldots, L-1), \\
P(z)=H(z) Q(z)-z^{-(M+1)} Q\left(z^{-1}\right) R(z),
\end{gathered}
$$

where $U(z)=\left(z^{-L} H\left(z^{-1}\right)\right) A(z)=\sum_{n=0}^{\infty} u_{n} z^{-n}$. In summary, $R(z)$ can be found by $H(z)$ and $Q(z)$ through (6). The optimal numerator polynomial $P(z)$ can be found analytically with a given $H(z), Q(z)$ and $R(z)$ through (7).

\section{B. Denominator calculation}

To calculate the location of poles, instead of extracting response characteristics through eigenvalue calculation in conventional methods, VISA determines $P(z)$ through an energyconserved allpass filter characteristic, denoted as the complementary signal [15]. First, the time-reversed response signal of the FIR filter $H(z)$ is defined as $\widetilde{H}[k]$, where $k=$ $0,1, \ldots L-1$. The idea of complementary signal is that if $\widetilde{H}[k]$ is fed into the allpass filter $A(z)$ of which the time response is $A[m], m=0,1, \ldots$, then the energy of the allpassfiltered time-reversed signal $a[n]=A[m] \otimes \widetilde{H}[k](\otimes$ denotes convolution) is distributed as

$$
\sum_{n=-\infty}^{\infty}|a[n]|^{2}=\sum_{n=-L+1}^{\infty}|a[n]|^{2}=\underbrace{\sum_{n=-L+1}^{0}|a[n]|^{2}}_{\Sigma_{1}}+\underbrace{\sum_{n=1}^{\infty}|a[n]|^{2}}_{\Sigma_{2}},
$$

where $\Sigma_{1}$ is the approximation error energy $E$ and $\Sigma_{2}$ is the energy of the approximant. The physical meaning is that, through an allpass filtering, an arbitrary signal can be separated into an error signal and an approximant signal in the time domain. The algorithm objective is to design an allpass filter which re-distributes the output energy of the filter in time domain and minimizes the signal energy within a time duration $0 \leq n \leq L-1$ (which is the error energy) $[9,15]$.

In numerical calculation, (5) is modified for designing an allpass operator $A^{(k)}(z)$ of a given order $M$ with

$$
\Delta^{(k)}(z)=A^{(k)}(z) z^{-1} R^{(k)}(z)=\frac{z^{-M} Q^{(k)}\left(z^{-1}\right)}{Q^{(k-1)}(z)} z^{-1} R^{(k)}(z) .
$$


Allpass operator (9) converges to an allpass function, if $\left\|Q^{(k)}(z)-Q^{(k-1)}(z)\right\| \rightarrow 0$, that is, the optimal denominator polynomial $Q(z)$ is found. Since $\left\|\Delta^{(k)}(z)\right\|=$ $\left\|R^{(k)}(z)\right\|=\left\|U^{(k)}(z)\right\|$ in (5) and (6), VISA involves a digital filtering operation (convolution) and a set of overdetermined equations to minimize $\left\|\Delta^{(k)}(z)\right\|$. First, we define $Q^{(0)}(z):=1, Q^{(k)}(z)=1+Q_{1}^{(k)}(z) z^{-1}, Q_{1}^{(k)}(z)=$ $\sum_{n=1}^{M-1} q^{(k)}(n) z^{-n}, X^{(k)}(z)=z^{-L} H\left(z^{-1}\right) / Q^{(k-1)}(z)=$ $\sum_{n=0}^{\infty} x^{(k)}(n) z^{-n}$, where $H(z)$ is the deconvoluted impulse response, $k$ is the number of iterations, and $Q_{1}^{(k)}(z)$ contains transient characteristics of the system and is used in the interpolation. By (5), (6) and (9), equation (10) is set up to solve the LS problem (11):

$$
\begin{aligned}
& U^{(k)}(z)=z^{-L} H\left(z^{-1}\right)\left(z^{-M} Q^{(k)}\left(z^{-1}\right) / Q^{(k-1)}(z)\right) \\
& \Rightarrow U^{(k)}(z)=X^{(k)}(z) z^{-M}\left(1+Q_{1}^{(k)}\left(z^{-1}\right)\right) \\
& \Rightarrow U^{(k)}(z)-z^{-M} X^{(k)}(z)=X^{(k)}(z) z^{-(M-1)} Q_{1}^{(k)}\left(z^{-1}\right),
\end{aligned}
$$

$\min \left\|\Delta^{(k)}(z)\right\|_{2}=\min \left\|\mathbf{U}^{(k)}(z)\right\|_{2}=\min \left\|\mathbf{B}^{(k)} \mathbf{q}^{(k)}-\mathbf{d}^{(k)}\right\|_{2}$,

where $\mathbf{q}^{(k)}=\left[\begin{array}{lll}q^{(k)}(M) & \ldots & q^{(k)}(1)\end{array}\right]^{T}$,

$\mathbf{d}^{(k)}=-\left[\begin{array}{llllll}0 & \cdots & 0 & x^{(k)}(0) & \cdots & x^{(k)}(L-M-1)\end{array}\right]^{T}$,

$\mathbf{B}^{(k)}=\left[\begin{array}{cccc}x^{(k)}(0) & 0 & \cdots & 0 \\ x^{(k)}(1) & x^{(k)}(0) & \ddots & \vdots \\ \vdots & \vdots & \ddots & 0 \\ x^{(k)}(M-1) & x^{(k)}(M-2) & \cdots & x^{(k)}(0) \\ \vdots & \vdots & & \vdots \\ x^{(k)}(L-1) & x^{(k)}(L-2) & \cdots & x^{(k)}(L-M)\end{array}\right]$.

The algorithm converges after sufficient iterations $N_{T}$, and we take $Q(z):=Q^{\left(N_{T}\right)}(z)$. By applying Cauchy-Schwarz inequality, it is proved that for an arbitrary $X^{(k)}(z)$ which minimizes (11), the maximum pole (zeros of $Q(z)$ ) radius $<1$, i.e., the macromodel is always stable [9], and the macromodel phase response will not be deteriorated by the pole flipping technique used in VF.

A discrete-time macromodel can be obtained from $P(z) / Q(z)$. The discrete-time macromodel can be used directly for frequency domain analysis or fixed time step simulation, transformed into an equivalent circuit [16], or into a continuous-time system by stability- and passivity-preserving bilinear transformation $\left(z=e^{s T} \approx(1+s T / 2) /(1-s T / 2)\right)$. VISA may not generate a passivity-guaranteed macromodel, but passivity enforcement techniques [17] can be applied.

Pseudocodes are given to summarize the flow of VISA:

1. Find $H(z)$, and $Q^{(1)}(z):=1$

2. Iterative pole calculation

2.1 Calculate $X^{(k)}(z)$ through a filtering (convolution) of $z^{-L} H\left(z^{-1}\right)$ with $Q^{(k)}(z)$

2.2 Construct $\mathbf{d}^{(k)}$ and $\mathbf{B}^{(k)}$ through elements of $X^{(k)}(z)$

2.3 Calculate the new $Q_{1}^{(k)}(z)$ and $R^{(k)}(z)$ by solving (11)

2.4 Repeat step 2.1 to step 2.3 until $Q_{1}^{(k)}(z)$ converges after
$N_{T}$ iterations

3. Calculate $P(z)$ through (6) and (7) with the given $H(z)$, $Q^{\left(N_{T}\right)}(z)$ and $R^{\left(N_{T}\right)}(z)$

\section{CONVERGENCE DISCUSSION OF VISA}

\section{A. VISA: Reformulation of Steiglitz-McBride iteration}

From (9), the objective function of VISA in each iteration becomes:

$$
\begin{aligned}
& \min \sum_{n=0}^{L-1}\left|\Delta^{(k)}\left(z_{n}\right)\right|^{2}=\min \sum_{n=0}^{M}\left|A^{(k)}\left(z_{n}\right) z^{-1} R^{(k)}\left(z_{n}\right)\right|^{2} \\
& =\min \sum_{n=0}^{L-1}\left|\frac{z^{-M} Q^{(k)}\left(z_{n}^{-1}\right)}{Q^{(k-1)}\left(z_{n}\right)} z^{-1} R^{(k)}\left(z_{n}\right)\right|^{2} \\
& =\min \sum_{n=0}^{L-1} \frac{1}{\left|Q^{(k-1)}\left(z_{n}\right)\right|^{2}}\left|z^{-(M+1)} Q^{(k)}\left(z_{n}^{-1}\right) R^{(k)}\left(z_{n}\right)\right|^{2} \\
& =\min \sum_{n=0}^{L-1} \frac{1}{\left|Q^{(k-1)}\left(z_{n}\right)\right|^{2}} \mid Q^{(k)}\left(z_{n}\right) H\left(z_{n}\right) \\
& \quad-\left.\left(Q^{(k)}\left(z_{n}\right) H\left(z_{n}\right)-z^{-(M+1)} Q^{(k)}\left(z_{n}^{-1}\right) R^{(k)}\left(z_{n}\right)\right)\right|^{2} \\
& =\min \sum_{n=0}^{L-1} \frac{1}{\left|Q^{(k-1)}\left(z_{n}\right)\right|^{2}}\left|Q^{(k)}\left(z_{n}\right) H\left(z_{n}\right)-P^{(k)}\left(z_{n}\right)\right|^{2} .
\end{aligned}
$$

Consequently, VISA is considered as a reformulation of the Steiglitz-McBride (SM) iteration [18]. In general, SM iteration converges to a near-global-optimal approximant in the LS sense for noise-free data.

\section{B. Model Order Selection}

As a reformulation of SM iteration, VISA provides a priori error bound is for an $M$ th-order approximant,

$$
\min _{\operatorname{deg}\left(\frac{P}{Q}\right)=M}\left(\frac{1}{2 \pi} \int_{-\pi}^{\pi}\left|H\left(e^{j \omega}\right)-\frac{P^{(k)}\left(e^{j \omega}\right)}{Q^{(k)}\left(e^{j \omega}\right)}\right|^{2} d \omega\right)^{1 / 2} \leq \sigma_{M+1},
$$

where $\sigma_{i}$ stands for the $i$ th Hankel singular value (HSVs). The macromodel order selection can be cast as an IIR filter order selection problem in our scenario. An upper triangular Hankel matrix $H$ is constructed and w.r.t a SISO system, $H$ reads

$$
H=\left[\begin{array}{cccc}
h_{1} & h_{2} & \cdots & h_{L} \\
h_{2} & h_{3} & \cdots & 0 \\
\vdots & \vdots & \ddots & \vdots \\
h_{L} & 0 & \cdots & 0
\end{array}\right]
$$

It is shown that the singular value of $H$ is equivalent to the Hankel singular values (HSV) of the impulse response system [19]. The HSVs can be computed efficiently and arranged in descending magnitude characterizing the order of importance of each state. Exactly analogous to balanced truncation [19], the macromodel order $M$ is chosen such that $\sigma_{M} \gg \sigma_{M+1}$. Such approach gives a quantitative metric to efficiently determine the value of an appropriate macromodel order. 


\section{Comparison with other algorithms}

As a simplification of SM iteration, VISA can be interpreted as a class of first-order (impulse) interpolation and secondorder (covariance) interpolation problem [14]. Comparing to the first-order interpolation (e.g., Padé approximation), SM iteration compensates the information of truncated responses (which always happens in the time-domain macromodeling) during approximation, generates stable macromodel and works robustly in noisy responses. Furthermore, unlike gradient methods, VISA converges to a near- $L_{2}$-optimal solution.

In numerical sense, conventional SM iteration suffers illconditioned calculation with hundreds of sampled data. VISA is superior to other SM-related algorithms since it uses polynomial basis instead of pole-based basis, does not require initial-pole assignment (since $Q^{(0)}(z):=1$ ), and its calculation is not deteriorated by the dynamic behavior and the initial-guess of pole-based basis. The numerator can be calculated through simple polynomial division, which does not involve any numerical-sensitive calculations (e.g., root finding techniques or numerical integration). However, we note that (11) may become ill-conditioned when the algorithm converges, which leads to $Q^{(k)}(z)$ with zeros (poles in the macromodel) in $|z| \geq 1$ and causes unexpected error. To alleviate the problem, techniques such as LSQR [20] can be used for illconditioned calculation of (11), using $Q^{(k-1)}(z)$ as an initial guess and a Givens orthogonalization pre-conditioner.

\section{D. $P$-norm approximation criteria}

The approximation framework (12) is generalized to a $P$ norm $\left(L_{p}\right)$ approximation, which suits different macromodeling requirements and gives a more realistic description of the system. For example, $L_{\infty}$ (Chebyshev norm) approximation gives a smaller macromodel for a linear-phase response, $L_{2}$ approximation gives a more accurate macromodel for a noisy response, and $L_{1}$ approximation is favorable for system identification with impulsive-noise-contaminated signals. For a $P$ norm approximation, the minimization framework (12) is generalized as

$$
\begin{aligned}
& \min \sum_{n=0}^{L-1}\left\|\Delta^{(k)}\left(z_{n}\right)\right\|_{p}=\min \left\|\mathbf{B}^{(k)} \mathbf{q}^{(k)}-\mathbf{d}^{(k)}\right\|_{p} \\
& =\min \sum_{n=0}^{L-1} \frac{1}{\| Q^{(k-1)}\left(z_{n}\right)}\left\|_{p}\right\| Q^{(k)}\left(z_{n}\right) H\left(z_{n}\right)-P^{(k)}\left(z_{n}\right) \|_{p},
\end{aligned}
$$

for which the over-determined equations of (15) can be solved using CVX [21] effectively. By applying CVX, the approximation framework can be generalized to a user-defined norm approximation, or (norm-)constrained approximation to meet different macromodeling requirements, e.g., passive rational interpolation. Furthermore, $P$-norm approximation may deteriorate the convergence property. $L_{\infty}$-constrained $L_{2}$ approximation can be used to improve the convergence performance.

\section{MIMO VISA EXTENSION}

VF-like algorithms require a large system equation to macromodel a multi-port system, so port splitting method is
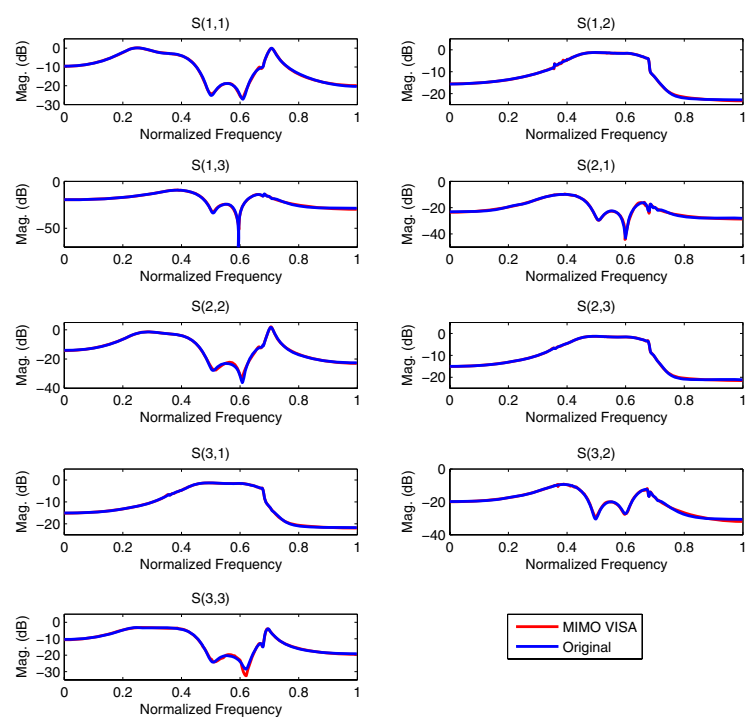

Fig. 1. Magnitude response of $\mathrm{S}(u, v)$ of the circulator example in normalized frequency domain. $\mathbf{S}(u, v)$ is the scattering parameter at input port $u$ and output port $v$.

applied to fit a subset of port responses at one time, which are then combined into a large macromodel. In the LS algorithm, the transfer matrix with a common denominator is used so that the Walsh's theorem can be applied to multi-port macromodeling. The optimal numerator polynomial can be calculated through (6) and (7) by replacing $H(z)$ in (7) by $H_{u, v}(z)$. The common denominator is calculated by the same basis function for all port responses, then all the elements of $\mathbf{B}_{\mathbf{p} \times \mathbf{q}}$ are stacked into a single column of over-determined equation:

$$
\begin{aligned}
& {\left[\begin{array}{llll}
\mathbf{B}_{1,1}^{(k)} & \mathbf{B}_{1,2}^{(k)} & \cdots & \mathbf{B}_{p, q}^{(k)}
\end{array}\right]^{T}\left[\mathbf{q}^{(k)}\right]} \\
& =\left[\begin{array}{llll}
\mathbf{d}_{1,1}^{(k)} & \mathbf{d}_{1,2}^{(k)} & \cdots & \mathbf{d}_{p, q}^{(k)}
\end{array}\right]^{T}
\end{aligned}
$$

where $\mathbf{B}_{u, v}^{(k)}$ and $\mathbf{d}_{u, v}^{(k)}$ are $\mathbf{B}$ and $\mathbf{d}$ in (11) for input port $u$ $(1 \leq u \leq p)$ and output port $v(1 \leq v \leq q)$, respectively. The model order selection criteria for the multiport macromodel can be developed based on the derivation in Section IVB. A significant advantage of MIMO VISA is that it has an $O\left(m^{2} L p q\right)$ complexity in each iteration in LS for denominator calculation of a multi-port system, whereas TD-VF has an $O\left((p q+1)^{2} m^{2} L p q\right)$ complexity for each iterative pole calculation, and other algorithms have a even higher computation complexity $[3,4]$. Hence, significant memory storage and computation time can be saved by using VISA.

\section{NUMERICAL EXAMPLES}

The proposed VISA is coded in Matlab m-script files and run in the Matlab 7.5 environment on a $1 \mathrm{~GB}-\mathrm{RAM} 3.4 \mathrm{GHz}$ PC. Examples are used to show the performance of VISA. 
TABLE I

COMPARISON BETWEEN VISA AND TD-VF IN THE CIRCULATOR EXAMPLE.

\begin{tabular}{c|c|c|c|c}
\hline & \multicolumn{2}{|c|}{ 25th order } & \multicolumn{2}{c}{ 32nd order } \\
\hline & VISA & TD-VF & VISA & TD-VF \\
\hline \hline $\begin{array}{c}\text { Max. deviation } \\
\text { when converged }\end{array}$ & 0.0019 & 0.0028 & 0.0011 & 0.0013 \\
$\begin{array}{c}\text { Avg. R.M.S. error } \\
\text { when converged } \\
\text { CPU Time for } \\
\text { convergence (sec.) } \\
\text { CPU Time to }\end{array}$ & 0.0069 & 0.0085 & 0.0055 & 0.0051 \\
$-40 \mathrm{~dB}$ error (sec.) & 2.17 & 40 & 4.29 & 44 \\
\hline
\end{tabular}

\section{A. Macrmodeling of three-port system responses}

The first example arises from a three-port counterclockwise $\mathrm{RF}$ circulator. Time-domain transient scattering responses are computed ranging to $4 \mathrm{GHz}$. All port responses (totally 9) are excited and fitted using MIMO VISA with a 25th-order macromodel. Time samples are taken at the intervals of $86 \mathrm{ps}$ for the first 800 points $(6.88 \mu \mathrm{s})$. The algorithm requires 13 iterations (4.07 seconds) to converge. Figs. 1 and 2 plot respectively the normalized frequency-domain responses and the time-domain responses of the converged approximant. Since there is a measurement defect at $0.68 f_{s}$ in the sampled data, VISA demonstrates robust and accurate fitting in both time and frequency domains. The model is also modeled using TD-VF [5] which is commonly used in commercial tools (IdEM [22]). The quantitative comparison of both algorithms is shown in Table I. It shows that VISA is an accurate (18\% less average R.M.S. error after convergence) and efficient algorithm $(>15 \times$ faster for convergence and $>17 \times$ faster to achieve a $-40 \mathrm{~dB}$ accuracy). The data is further fitted using MIMO VISA and TD-VF with a 32nd-order macromodel. From Table I, MIMO VISA again generates a more accurate macromodel in the $L_{\infty}$ sense and with more efficient computation. Fig. 3 shows the $L_{2}$ error during iterations for the 25th-order and 32nd-order macromodels, showing that VISA converges faster than TD-VF. In general, VISA converges quickly (within 60 iterations), and especially for minimum-phase response.

\section{B. Algorithm performance analysis}

Next, we investigate the use of HSVs in guiding the model order selection in VISA. Computing HSVs requires $58 \mathrm{sec}-$ onds, and Fig. 4(a) shows the HSVs of the impulse response of the circulator example. The figure shows a significant drop of HSVs at first and then gradually afterwards. Region of largest HSVs and the relative error of different macromodel orders are shown in Fig. 4(b). Finally, we study the robustness of VISA. First, LSQR with initial guess and Givens orthogonalization pre-conditioner is adopted to solve (16) to explore the effectiveness of handling ill-conditioned case. VISA generates a 25th-order macromodel within 7.1 seconds, with $0.0081 L_{2}$ error and $0.0024 L_{\infty}$ error. Also, we repeat the circulator example with a 25th-order model under an SNR of 30dB. In this case, VISA converges with a $-33.5 \mathrm{~dB}$ error.
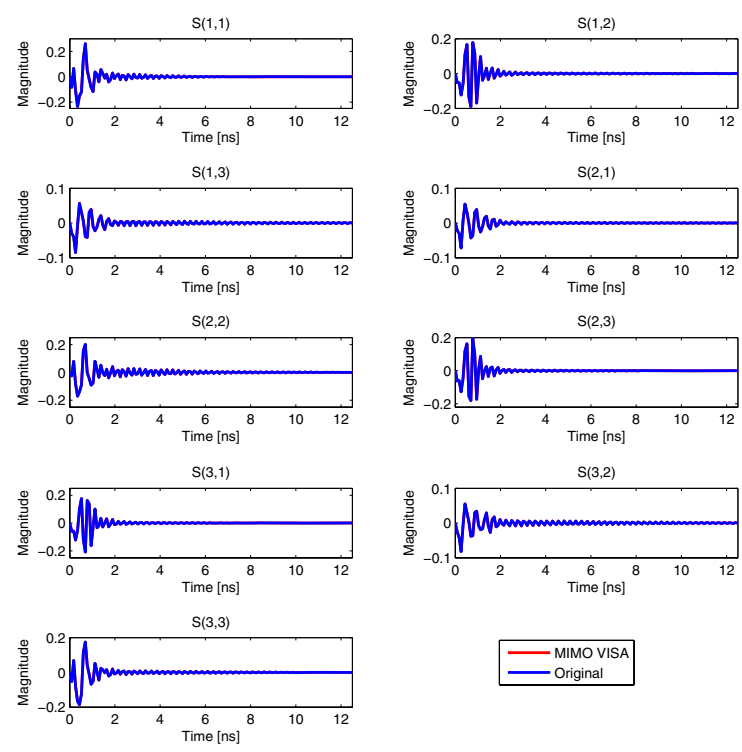

Fig. 2. Time response of $\mathbf{S}(u, v)$ of the circulator example.

TABLE II

COMPARISON BETWEEN DIFFERENT $P$-NORM APPROXIMATION, WHERE $2^{*}$ REPRESENTS $L_{\infty}$-CONSTRAINED 2-NORM APPROXIMATION.

\begin{tabular}{c|c|c|c|c}
\hline$P$-norm approx. & $L_{1}$ err. & $L_{2}$ err. & $L_{\infty}$ err. & CPU Time (sec.) \\
\hline \hline 1 & 0.0932 & 0.0111 & 0.0042 & 213.67 \\
$2(\mathrm{CVX})$ & 0.0674 & 0.0028 & $4.4 \mathrm{e}-4$ & 4.44 \\
$\infty$ & 0.0664 & 0.0027 & $3.1 \mathrm{e}-4$ & 7.05 \\
\hline $2^{*}$ & 0.0657 & 0.0027 & $2.7 \mathrm{e}-4$ & 3.35 \\
$2(\mathrm{QR})$ & 0.0674 & 0.0028 & $4.4 \mathrm{e}-4$ & 0.11 \\
\hline \hline
\end{tabular}

\section{C. $P$-norm approximation}

Response $\mathrm{S}(1,1)$ has been extracted and fitted using 1-norm, 2-norm using CVX (LS-CVX), $L_{\infty}$-constrained 2-norm, 2norm using QR decomposition (LS-QR), and $\infty$-norm approximation, respectively, with a 13th-order SISO macromodel, and they converge within $30,10,3,10$ and 20 iterations, respectively, where CVX and QR denote solving using CVX and QR decomposition, respectively. The implementation details are shown in Table II. It shows approximation can be more accurate using a non-traditional norm selection $\left(3.5 \% L_{2}\right.$ error, $29.5 \% L_{\infty}$ error and $24.5 \% \mathrm{CPU}$ time reduction, comparing to LS-CVX approximation). Comparing with original approach, $P$-norm approximation can give a more accurate solution, at the expense of more computation time.

\section{Macromodeling of benchmark examples}

The four benchmark examples in [23] arise from the macromodeling of measured two-port time-responses of an on-chip resistor (RPOLY2-ME), an inductor (SP-SMALL), a coplanar line (U-COPL) and a capacitor (CMIM). TD-VF cannot give a converged result for the first three examples, whereas VISA produces converged solutions for all the examples. The quan- 

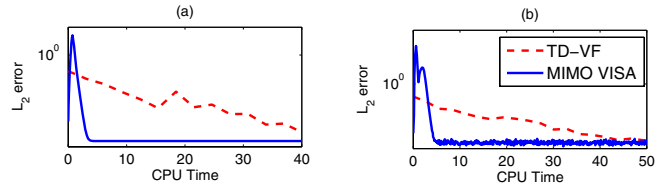

Fig. 3. $L_{2}$ error in macromodeling using TD-VF and VISA in the circulator example. (a) 25th-order macromodel and (b) 32nd-order macromodel.
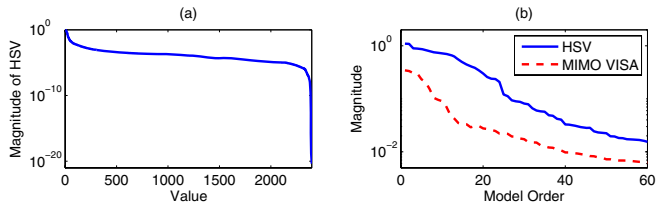

Fig. 4. Hankel singular values of the system in the circulator example. (a) Entire HSVs and (b) Comparison with the macromodel error.

titative comparison of the best result of TD-VF and the converged result of VISA is shown in Table III, generating macromodel with $51 \%$ less $L_{2}$ error on average and much faster computation. These promising results demonstrate the superiority of VISA in time-domain macromodeling.

\section{CONCLUSION}

VISA has been presented for time-domain macromodeling to generate discrete-domain macromodels. It has been shown that VISA constitutes a simplified MIMO Steiglitz-McBride iteration without pole-sensitive computations. Model order selection and $P$-norm approximation have been proposed for facilitating the macromodeling process. Practical examples have confirmed that VISA exhibits efficient and accurate computation when compared to commercial TD-VF algorithms.

\section{ACKNOWLEDGMENT}

This work was supported in part by The Research Grants Council (RGC) GRF grants HKU 718509E and HKU 717407E.

\section{REFERENCES}

[1] R. Achar and M. S. Nakhla, "Simulation of high-speed interconnects," Proc. IEEE, vol. 89, no. 5, pp. 693-728, May 2001.

TABLE III

COMPARISON BETWEEN VISA AND TD-VF IN THE BENCHMARK EXAMPLES. THE BRACKETED VALUE IS THE TD-VF RESULT.

\begin{tabular}{c|c|c|c}
\hline & $\begin{array}{c}\text { Max. dev- } \\
\text { iation }\end{array}$ & $\begin{array}{c}\text { Avg. R.M.S. } \\
\text { error }\end{array}$ & $\begin{array}{c}\text { CPU } \\
\text { (sec. })\end{array}$ \\
\hline \hline RPOLY2-ME & $0.0002(0.0004)$ & $0.0006(0.0008)$ & $0.24(8)$ \\
CMIM & $0.0001(0.0032)$ & $0.0005(0.0149)$ & $0.40(8)$ \\
SP-SMALL & $0.0003(0.0008)$ & $0.0007(0.0027)$ & $0.63(8)$ \\
U-COPL & $0.0003(0.0004)$ & $0.0010(0.0011)$ & $0.21(7)$ \\
\hline \hline
\end{tabular}

[2] B. Gustavsen and A. Semlyen, "Rational approximation of frequency domain responses by vector fitting," IEEE Trans. Power Delivery, vol. 14, no. 3, pp. 1052-1061, Jul. 1999.

[3] I. Munteanu and D. Ioan, "Parameter extraction for microwave devices based on 4SID techniques," IEEE Trans. Magn., vol. 35, no. 3, pp. 17811784, May 1999.

[4] Y. Hua and T. K. Sarkar, "Generalized pencil-of-function method for extracting poles of an EM system from its transient response," IEEE Trans. Antennas Propagat., vol. 37, no. 2, pp. 229-234, Feb. 1999.

[5] S. Grivet-Talocia, "Package macromodeling via time-domain vector fitting," IEEE Microwave Guided Wave Lett., vol. 13, no. 11, pp. 472-474, Nov. 2003.

[6] S. Grivet-Talocia, F. G. Canavero, I. S. Stievano, and I. A. Maio, "Circuit extraction via time-domain vector fitting," in Proc. Int. Symp. Electromagnetic Compatibility, Aug. 2004, pp. 1005-1010.

[7] C. U. Lei and N. Wong, "Efficient linear macromodeling via discretetime time-domain vector fitting," in Proc. Intl. Conf. on VLSI Design, Jan. 2008, pp. 469-474.

[8] C. U. Lei, H. K. Kwan, Y. Liu, and N. Wong, "Efficient linear macromodeling via least-squares response approximation," in Proc. IEEE Symp. Circuits and Systems, May 2008, pp. 2993-2996.

[9] H. Brandenstein and R. Unbehauen, "Least-squares approximation of FIR by IIR digital filters," IEEE Trans. Signal Processing, vol. 46, no. 1, pp. 21-30, Jan. 1998.

[10] N. Wong and C. U. Lei, "IIR approximation of FIR filters via discretetime vector fitting," IEEE Trans. Signal Processing, vol. 56, no. 3, pp. 1296-1302, Mar. 2008.

[11] C. U. Lei and N. Wong, "IIR approximation of FIR filter via discretetime hybrid-domain vector fitting," IEEE Signal Process. Lett., vol. 16, no. 6, pp. 533-537, Jun. 2009.

[12] Y. S. Mekonnen and J. E. Schutt-Aine, "Fast broadband macromodeling technique of sampled time/frequency data using z-domain vector-fitting method," in Proc. IEEE Electronic Components and Tech. Conf., May 2008, pp. 1231-1235.

[13] J. L. Walsh, Interpolation and Approximation by Rational Functions in the Complex Domain Providence. RI: Amer. Math. Soc., 1965.

[14] P. A. Regalia, M. Mboup, and M. Ashari, "A class of first- and secondorder interpolation problems in model reduction," AEU International Journal of Electronics and Communication, vol. 49, pp. 332-343, 1995.

[15] T. Young and W. Huggins, "'Complementary' signals and orthogonalized exponentials," IRE Trans. on Circuit Theory, vol. 8, no. 4, pp. 362370, Dec. 1962.

[16] L. N. et al., "z-transform-based methods for electromagnetic transient simulations," IEEE Trans. Power Delivery, vol. 22, no. 3, pp. 17991805, Jul. 2007.

[17] S. Grivet-Talocia and A. Ubolli, "A comparative study of passivity enforcement schemes for linear lumped macromodels," IEEE Trans. Adv. Packag., vol. 31, no. 4, pp. 673-683, Nov. 2008.

[18] K. Steiglitza and L. McBride, "A technique for the identification of linear systems," IEEE Trans. Automat. Contr., vol. 10, no. 4, pp. 461-464, Oct. 1965.

[19] G. Gu, "All optimal hankel-norm approximations and their $l_{\text {inf }}$ error bounds in discrete-time," International Journal of Control, vol. 78, no. 6, pp. 408-423, Apr. 2005.

[20] C. C. Paige and M. A. Saunders, "LSQR: An algorithm for sparse linear equations and sparse least squares," ACM Trans. Math. Soft., vol. 8, pp. 43-71, 1982.

[21] M. Grant and S. Boyd, "CVX: Matlab software for disciplined convex programming." [Online]. Available: http://stanford.edu/ boyd/cvx/

[22] "Official website of IdemWorks." [Online]. Available: http://www.idemworks.com/

[23] D. Ioan and G. Ciuprina, "Reduced order models of on-chip passive components and interconnects, workbench and test structures," in Model Order Reduction: Theory, Research Aspects and Applications. Springer, 2008, pp. 447-467. 